\title{
Adaptive Sliding Mode Control of Airship Pitch Channel Attitude Angle
}

\author{
Guoqiang Liang ${ }^{1}$, Guangbin $\mathrm{Wu}^{1}$, Lingling Wang ${ }^{1}$ and Junwei Lei ${ }^{1, \text { a }}$
}

${ }^{1}$ Department of control engineering, Naval aeronautical and astronautical University, Yanti, 264001

aleijunwei@126.com

Keywords: airship; pitch channel; adaptive; robustness; stability

\begin{abstract}
On the basic of nonlinear model of pitching channel of airship, assuming the condition that the information of the unknown portion of the assumed airship model satisfy the norm-bounded condition and the boundary is known. A adaptation method has been used to solve the unknown information problem in this paper, and this paper has proposed a class of sliding mode control method in order to control attitude angle of pitching channel of airship. Finally, this control method is proved to be effective by numerical simulation.
\end{abstract}

\section{Introduction}

As a aerial platform, airship has unique advantage, such as low cost, long hang time, large load ability, small noise, low power consumption, excellent security, high cost-effectiveness and so on.

But there is a lot of information about the design of airship and stability analysis, and there is little information about the control system of airship for secrecy reason.

In 1998, Brazil AURORA project team of stratospheric airship published initial literature[1]. Literature[1] has given YEZ-2A airship's six degree-of-freedom model that has been confirmed by test flight, and literature[2] has analysed the part of dynamic response.

On the basic of six degree-of-freedom model of airship, this paper extracts the simplified nonlinear model of pitching channel for the research about airship control. Because the airship model has its irresistible inaccuracy or unknown, but the inaccuracy or unknown satisfy he norm-bounded condition and the boundary is unknown, this paper proposes a control that combines self-adaptation and sliding mode control to control airship attitude. Finally, this control method is proved to be effective by numerical simulation.

\section{Model Description}

Based on the previous work, the pitch channel model of airship can be described as follows:

$$
M \dot{x}=f(x)+g(x) u
$$

And $x=\left[\begin{array}{llllll}u & w & q & \theta & x & z\end{array}\right], M$ satisfies

$$
M^{-1}=\left[\begin{array}{llllll}
a_{11} & & a_{13} & & & \\
& a_{22} & & & & \\
a_{31} & & a_{33} & & & \\
& & & 1 & & \\
& & & & 1 & \\
& & & & & 1
\end{array}\right]
$$

The definition of $a_{i j}$ see the definition of $M$ in previous work[3-5].

Choose the expect value of all states $u, w, q, \theta, x, z$ are $u^{d}, w^{d}, q^{d}, \theta^{d}, x^{d}, z^{d}$,Define the error variable $e=x-x^{d}, \dot{e}=\dot{x}$, then it hold

Use the inverse matrix of $M$

$$
M \dot{e}=f(x)+g(x) u
$$




$$
\dot{e}=M^{-1} f(x)+M^{-1} g(x) u
$$

To make it convenient for reading, some functions can be written as follows[6-8]

$$
f(x)=\left[\begin{array}{l}
f_{1} \\
f_{2} \\
f_{3} \\
f_{4} \\
f_{5} \\
f_{6}
\end{array}\right], \quad g(x)=\left[\begin{array}{cc}
0 & 1 \\
k_{1} & 0 \\
k_{2} & 0 \\
0 & 0 \\
0 & 0 \\
0 & 0
\end{array}\right], \quad u=\left[\begin{array}{ll}
u_{1} & u_{2}
\end{array}\right]^{T}
$$

where

$$
\left[\begin{array}{l}
f_{1} \\
f_{2} \\
f_{3} \\
f_{4} \\
f_{5} \\
f_{6}
\end{array}\right]=\left[\begin{array}{c}
-\left(m+m_{33}\right) w q+Q\left[C_{X 1} \cos ^{2} \alpha+C_{X 2} \sin (2 \alpha) \sin (\alpha / 2)\right. \\
\left(m+m_{11}\right) q u+m a_{z} q^{2}+Q\left[C_{z 1} \cos (\alpha / 2) \sin (2 \alpha)+C_{z 2} \sin (2 \alpha)+C_{z 3} \sin (\alpha) \sin (\mid \alpha)\right] \\
-m a_{z} w q(-r v)+Q\left[C_{M 1} \cos (\alpha / 2) \sin (2 \alpha)+C_{M 2} \sin (2 \alpha)+C_{M 3} \sin (\alpha) \sin (\alpha)\right]-a_{z} \sin \theta W \\
q \\
u \cos \theta+w \sin \theta \\
-u \sin \theta+w \cos \theta
\end{array}\right]
$$

Define[9]

And

$$
M^{-1} f(x)=\left[\begin{array}{c}
f_{a 1} \\
f_{a 2} \\
f_{a 3} \\
f_{a 4} \\
f_{a 5} \\
f_{a 6}
\end{array}\right]=\left[\begin{array}{c}
a_{11} f_{1}+a_{13} f_{3} \\
a_{22} f_{2} \\
a_{31} f_{1}+a_{33} f_{3} \\
f_{4} \\
f_{5} \\
f_{6}
\end{array}\right]
$$

Then the system can be written as follows

$$
g(x) u=\left[\begin{array}{c}
u_{2} \\
k_{1} u_{1} \\
k_{2} u_{1} \\
0 \\
0 \\
0
\end{array}\right]
$$

$$
\left[\begin{array}{c}
\dot{u} \\
\dot{w} \\
\dot{q} \\
\dot{\theta} \\
\dot{x} \\
\dot{z}
\end{array}\right]=\left[\begin{array}{c}
f_{a 1} \\
f_{a 2} \\
f_{a 3} \\
f_{a 4} \\
f_{a 5} \\
f_{a 6}
\end{array}\right]+\left[\begin{array}{c}
a_{11} u_{2}+a_{13} k_{2} u_{1} \\
a_{22} k_{1} u_{1} \\
a_{31} u_{2}+a_{33} k_{2} u_{1} \\
0 \\
0 \\
0
\end{array}\right]
$$

\section{Robust Sliding Mode Control of Attitude}

Assume that the airship moves at constant speed and attitude, and assume angle of pitch $\theta^{d}=2 / 57.3$, then define sliding mode surface[10-11]:

$$
s_{1}=c_{1}\left(\theta-\theta^{d}\right)+q
$$


Differentiate the sliding mode surface

$$
\dot{s}_{1}=c_{1} q+\dot{q}=c_{1} q+a_{31} f_{1}+a_{33} f_{3}+a_{31} u_{2}+a_{33} k_{2} u_{1}
$$

Consider decoupling control, $u_{1}$ controls vertical movement and $u_{2}$ controls forward movement, then design:

$$
u_{2}=\text { Cons }
$$

Assume $a_{31} f_{1}+a_{33} f_{3}$ is bounded and the boundary is known, then[12-15]:

$$
a_{31} f_{1}+a_{33} f_{3}<d_{1} a_{33} k_{2}
$$

If the boundary is unknown, choose the value of the boundary $\hat{d}_{1}$, the design control law as follow:

$$
u_{1}=u_{1 b}=-k_{0} s_{1}-\hat{k}_{1} s_{1}-\hat{k}_{2} q-\hat{d}_{1} \operatorname{sign}\left(s_{1}\right)-\hat{k}_{4} u_{2}
$$

then

$$
\begin{aligned}
s_{1} \dot{s}_{1} \leq & -a_{1} s_{1} s_{1}+\left(c_{1}-a_{33} k_{2} \hat{k}_{2}\right) q s_{1}+\left(a_{31} f_{1}+a_{33} f_{3}-a_{33} k_{2} \hat{d}_{1}\right) s_{1} \\
& +\left(a_{31}-a_{33} k_{2} \hat{k}_{4}\right) u_{2} s_{1}+\left(a_{1}-a_{33} k_{2} k_{0}-a_{33} k_{2} \hat{k}_{1}\right) s_{1} s_{1}
\end{aligned}
$$

It can be got by simplifying

$$
\begin{aligned}
s_{1} \dot{s}_{1} \leq & -a_{1} s_{1} s_{1}+\left(c_{1}-a_{33} k_{2} \hat{k}_{2}\right) q s_{1}+d_{1}\left|s_{1}\right|-a_{33} k_{2} \hat{d}_{1}\left|s_{1}\right| \\
& +\left(a_{31}-a_{33} k_{2} \hat{k}_{4}\right) u_{2} s_{1}+\left(a_{1}-a_{33} k_{2} k_{0}-a_{33} k_{2} \hat{k}_{1}\right) s_{1} s_{1}
\end{aligned}
$$

Define:

$$
\tilde{d}_{1}=d_{1}-a_{33} k_{2} \hat{d}_{1}
$$

then

$$
\dot{\tilde{d}}_{1}=-a_{33} k_{2} \dot{\hat{d}}_{1}
$$

Where

$$
\dot{\hat{d}}_{1}=\Gamma_{d} S_{1}
$$

So

$$
\left[\frac{1}{2 \Gamma_{d} a_{33} k_{2}}(\tilde{d})^{2}\right]^{\prime}=-\tilde{d} s_{1}
$$

choose a Lyapunov function as[16-19]:

$$
V_{c}=\frac{1}{2} s_{1}^{2}+\sum_{i=1, i \neq 3}^{4}\left[\frac{1}{2 \Gamma_{i} a_{33} k_{2}}\left(\tilde{k_{i}}\right)^{2}\right]+\frac{1}{2 \Gamma_{i} a_{33} k_{2}}(\tilde{d})^{2}
$$

So

$$
\dot{V}_{c} \leq-a_{1} s_{1}^{2} \leq 0
$$

It is not difficult to prove that system is stable in hypothetical condition.

\section{Numerical Simulation}

The system is proved to be stable by theoretical derivation as above, in order to test the stability of the system, this section uses SIMULINK tool case in MATLAB to the simulation.

In this section, choose $u_{2}=10000$, assume that the initial height is 1 meter, assume that attitude angle is 20 degree, and choose $d_{1}=0.01$, simulation results are as follows: 


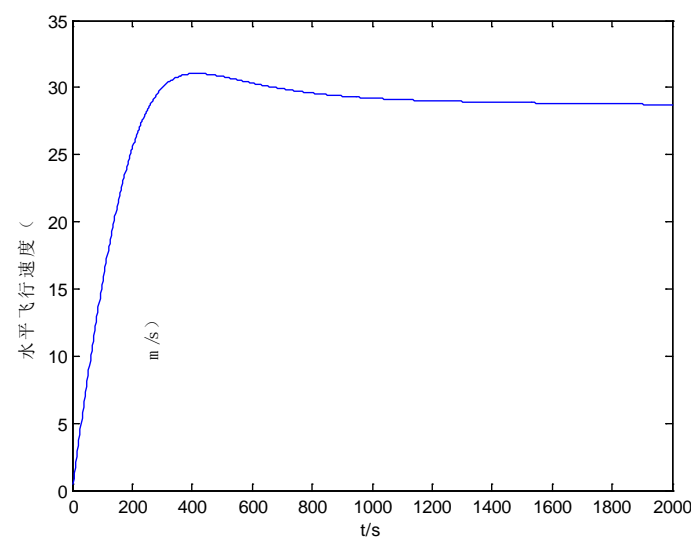

Fig. 1 Forward Velocity

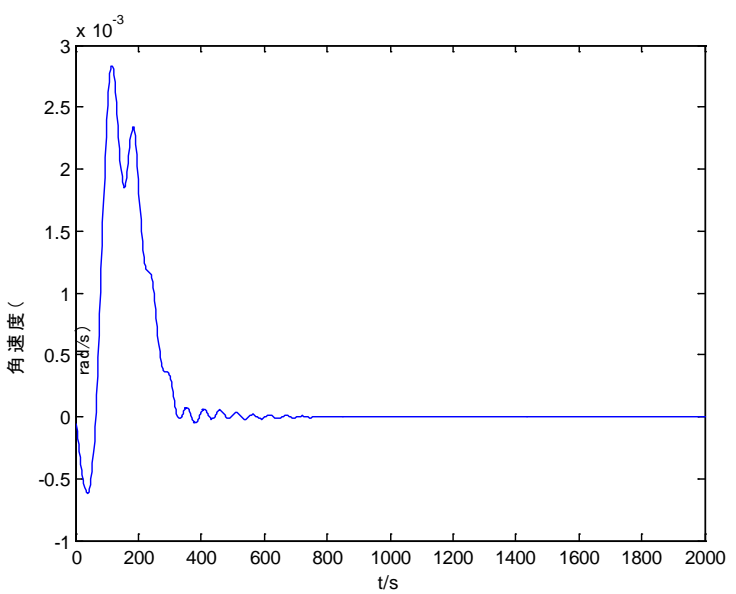

Fig. 3 Angle Velocity

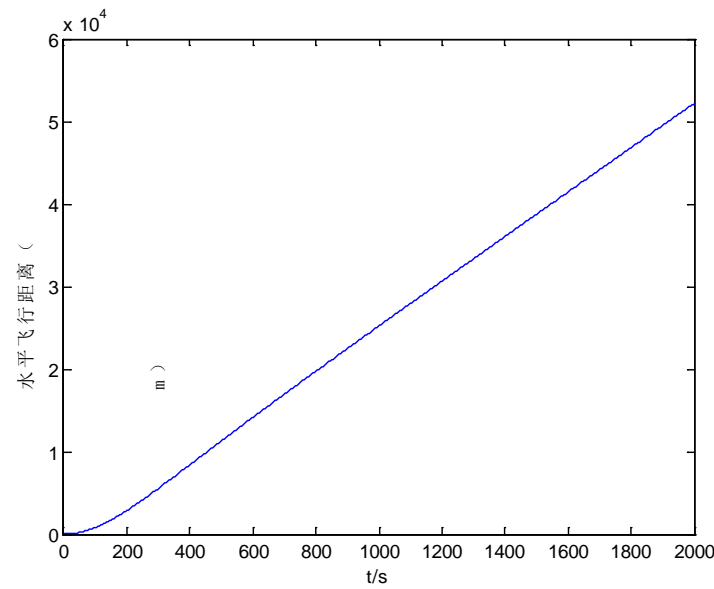

Fig. 5 Flying Distance

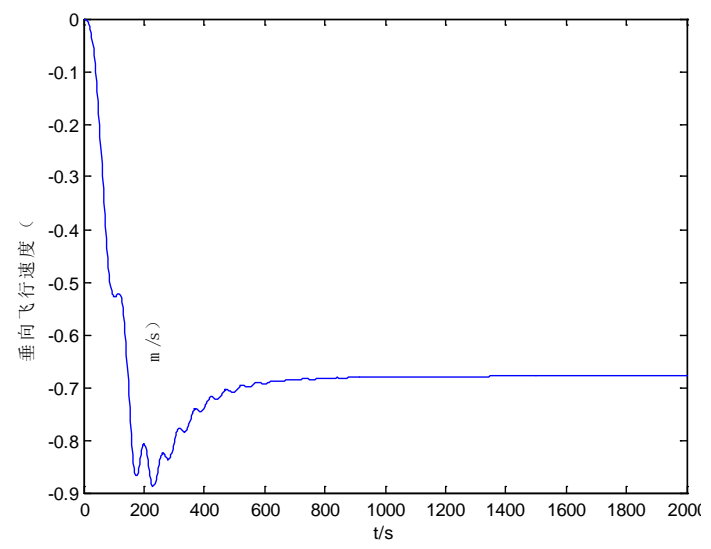

Fig. 2 Vertical Velocity

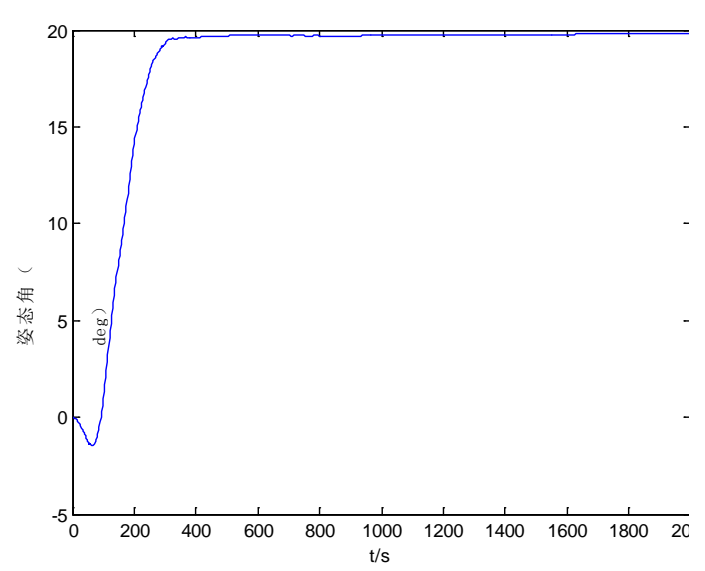

Fig. 4 Pitch Angle

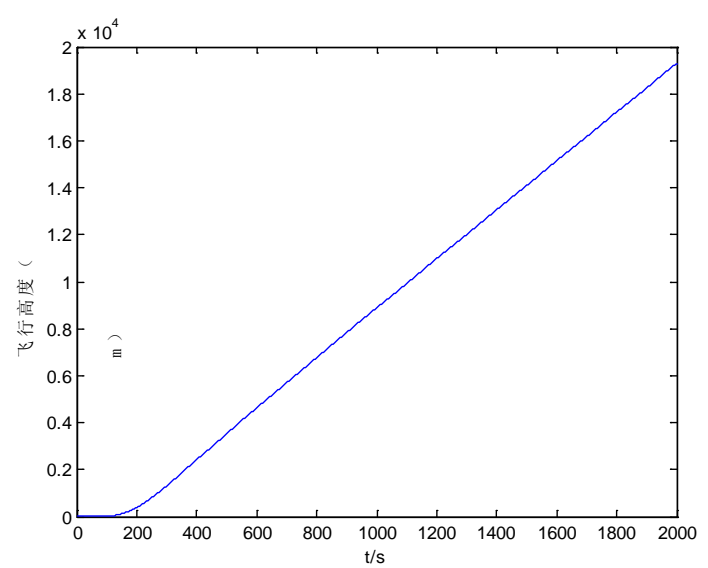

Fig. 6 Height 


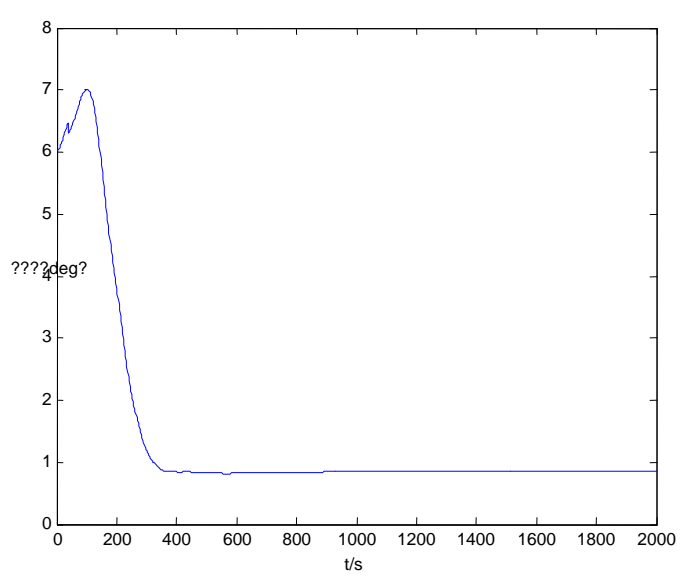

Fig. 7 Actuator Angle

\section{Conclusion}

This paper proposes a control that combines self-adaptation and sliding mode control to solve problem that some information in nonlinear model of pitching channel of airship are unknown. On the basic of nonlinear model of pitching channel of airship, this paper proposes a control that combines self-adaptation and sliding mode control in hypothetical condition that the information of the unknown portion of the system satisfy the norm-bounded condition. The simulation results show that the vibration chatter of system and offset of attitude angle is alleviated and the gain of controller don't need to be raise. So the control method preposed in this paper is better than smooth function from the point of alleviating offset.

\section{References}

[1] Elfes A., S.Bergerman, M.Ramos. A semi-autonomous robotic airship for environmental monitoring missions. IEEE International Conference on Robotics and Automation. 1998. Leuven, Belgium.

[2] Gomes S B V, Romos J J G. Airship Dynamic Modeling for Autonomous Operation. Proceedings of the 1998 IEEE International Conference on Robotics and Automation. 1998, 3462-3467.

[3] L.B.Tuckerman.Inertia Factors of Ellipsoids for Use in Airship Design. Naca Reports.2006, 14(3):45 50

[4] E.C.de Paiva,S.S.Bueno,Influence of Wind Speed on Airship Dynamics, Journal of Guidance,Control and Dynamics.2002,25(6):116 124

[5] Sergio B.Varella Gomes and Josue Jr.G.Ramos.Airship dynamic modeling for autonomous operation.Proceedings of the 2003 IEEE. International Conference on Robotics\&Automation. 2003:5 14

[6] J.S.Uhlman,N.E.Fine,D.C.Kring.Calculation of the Added Mass and Damping Forces on Supercavitating Bodies.The 4th International Symposium on Cavitation,California,2001:7 13

[7] D.Clarke.Calculation of the Added Mass of Elliptical Cylinders in Shallow Water.Ocean Engineering.2001,28(4):61 72

[8] C.J.Atkinson,R.G.Urso.Modeling of Apparent Mass Effects for the Real-Time Simulation of a Hybird Airship.AIAA Modeling and Simulation Technologies Conference and Exhibit,Keystone.2006:21 32

[9] Yokomaku Y.The Stratospheric Platform Airship R\&D Program of Japan. The 2nd Stratospheric 
Platform Systems Workshop,Tokyo Japan.2000:7 13

[10] S.P.Jones,J.D.Laurier.Aerodynamic Estimation Techniques for Aerostats and Airships.AIAA Lighter-than-Air Systems Conference,Annapolis,2004: 88 94

[11]M.T.Soylemeza,N.Munrob,H.Bakic.Fast Calculation of Stabilizing PID Controllers.Automatic,2003,39(7):121 126

[12] Etkin B, Theory of the flight of Airplanes in Isotropic Turbulence Review an Extension,AGARD Rept.1961:372

[13] David K. Schmidt,James Stevens, Jason Roney.Dynamic Modeling,Control, and Station-Keeping Guidance of A Large High-Altitude“Near-Space” Airship.AIAA Guidance,Navigation,and Control Conference and Exhibit. 2006-6781 